\title{
Taxotere Induces Dephosphorylation of MET in Patient-derived Tumor Models
}

\author{
HYEMIN MUN ${ }^{1 *}$, SUN-HYE LEE ${ }^{1 *}$, CHU-HEE LEE ${ }^{2 *}$, SE-YOUNG JO $^{1}$, \\ JU-HEE OH ${ }^{1}$, AREUM LEE ${ }^{1}$, BORA LEE ${ }^{1}$, SE JIN JANG ${ }^{1}$ and YOUNG-AH SUH ${ }^{1}$ \\ ${ }^{1}$ Department of Biomedical Sciences, The University of Ulsan College of Medicine, \\ Asan Medical Center, Seoul, Republic of Korea; \\ ${ }^{2}$ Department of Biochemistry and Molecular Biology, School of Medicine, \\ Yeungnam University, Daegu, Republic of Korea
}

\begin{abstract}
Background/Aim: Although molecular targeting therapy is an attractive treatment for cancer, resistance eventually develops in most cases. Here, we evaluated chemotherapeutic efficacy on non-small cell lung cancer (NSCLC) with acquired resistance to epidermal growth factor receptor inhibitors mechanistically. Materials and Methods: Antitumor effects of taxotere were evaluated using multiple models, including xenograft, and patient-derived models developed from adenocarcinoma cancer patients. Protein expressions were analyzed after drug treatment. Results: Taxotere inhibited tumor growth of NSCLC cells harboring drug resistance, and reduced the expression of phosphorylated MET proto-oncogene, receptor tyrosine kinase (MET). A tumor-inhibitory effect of taxotere was also demonstrated in vivo in xenografts in mice, patient-derived primary lung tumor cells and patient-derived xenograft with concomitant repression of phosphorylated MET expression. Chemotherapeutic and MET-targeting drug exhibited a synergistic cell growthinhibitory effect. Conclusion: These results suggest that the anticancer drug taxane may be an adjuvant for lung tumors exhibiting enhanced signaling of MET networks.
\end{abstract}

Many targeted cancer therapeutics have opened up the era of precision medicine (1) through advances of biological techniques, including the development of models resembling patient tumors (2). Patients diagnosed with non-small cell

*These Authors contributed equally to this study.

Correspondence to: Young-Ah Suh, Ph.D., Department of Biomedical Sciences, The University of Ulsan College of Medicine, Asan Medical Center, Seoul, Republic of Korea. Tel: +82 230102646, Fax: +82 230102630, e-mail: ysuh@amc.seoul.kr

Key Words: EGFR inhibitor resistance, patient-derived model, MET signaling, combinational treatment, tumor microenvironment. lung cancer (NSCLC) have benefits from specific 'targeted' anticancer drugs. In practice, resistance mechanisms often develop to most of these targeted agents, however, and patients need alternative treatments (3). Together with discovering new agents that can combat acquired resistance, standard chemotherapeutics as well as combinational adjuvants are being re-evaluated to overcome resistance in order to increase survival rates.

Several mechanisms have been elucidated for acquired resistance to epidermal growth factor receptor (EGFR) inhibitors (4-6). Approximately $40 \%$ of patients who were initially sensitive to tyrosine kinase inhibitor (TKI) therapy develop secondary mutation of the T790M at exon 20 in EGFR. Overexpression of hepatocyte growth factor (HGF) receptor has also been observed in both TKI-naive patients and those with acquired resistance to EGFR inhibitors through amplification of the MET gene in approximately $20 \%$ of acquired resistance patients to EGFR inhibitors (7, 8). Therefore, antibodies targeting MET and HGF, as well as small molecule kinase inhibitors, have been investigated in preclinical and clinical trials $(9,10)$.

Recently, increasing evidence has revealed the importance of immunotherapy and the tumor microenvironment, and has led to the development of immune checkpoint inhibitors (11). Fast-growing tumor cells establish contact with stromal cells via a complicated intercellular signaling network, in which chemokines and cytokines play critical roles (12).

In this regard, we validated the antitumor effect of taxotere, a standard chemotherapeutic (13), on different cells and mouse models.

\section{Materials and Methods}

Cell culture and reagents. Human lung cancer cell line H1975 was obtained from the American Type Culture Collection (Manassas, VA, USA) and maintained in Dulbecco's modified Eagle's medium or RPMI-1640 medium (GIBCO BRL, Carlsbad, CA, USA) containing $10 \%$ fetal bovine serum (GIBCO BRL), $100 \mathrm{U}$ penicillin, 
Table I. Primers used for reverse transcription polymerase chain reaction.

\begin{tabular}{llll}
\hline Gene symbol & Encoded protein & Primer sequence \\
\hline CX3CL1 & & Forward & GACCCCTAAGGCTGAGGAAC \\
& & Reverse & CTCTCCTGCCATCTTTCGAG \\
CCL2 & C-C motif chemokine ligand 2 & Forward & TGGAATCACCTGCTGTTAT \\
& & Reverse & AACTCCGAAACAATTGTACTCAGCTG \\
CCL26 & C-C Motif chemokine ligand 26 & Forward & GTAACTCTGGGAGGAAACACCCTCTCC \\
& & Reverse & TCAATGAGGAGACTTGCCTG \\
IL6 & Interleukin 6 & Forward & TCATCTGCACAGCTCTGGCT \\
& & Reverse & ATGACTTCCAAGCTGGCCGTGGCT \\
IL8 & Interleukin 8 & Forward & TCTCAGCCCTCTTCAAAAACTTCT \\
& & Reverse & GGCCATTACTACGACATCCTG \\
ICAM1 & Serpin family E member 1 & Forward & GGTCATGTTGCCTTCCAGT \\
& & Reverse & GGAAGGTGTATGAACTGAGCAA \\
GAPDH & Intercellular adhesion molecule 1 & Forward & GGAGTCCAGTACACGGTGA \\
& & Reverse & TCACCACCATGGAGAAGGC \\
& Glyceraldehyde 3-phosphate dehydrogenase & Forward & GCTAAGCAGTTGGTGGTCA \\
\hline
\end{tabular}

and $100 \mu \mathrm{g} / \mathrm{ml}$ streptomycin at $37^{\circ} \mathrm{C}$ in $5 \% \mathrm{CO}_{2}$. All cell lines routinely tested negative for mycoplasma (PlasmoTest; InvivoGen, San Diego, CA, USA). To verify cell viability, a trypan blue exclusion assay was routinely performed. Cells were counted with an Automated Cell Counter (BioRad, Hercules, CA, USA). Taxotere was purchased from Chemie Tek (Indianapolis, IN, USA) and crizotinib, a specific MET inhibitor, was purchased from Selleck Chemicals LLC (Houston TX, USA).

Protein expression analysis. Western blot analysis was performed as described previously. Total cellular proteins $(20 \mu \mathrm{g})$ were subjected to analysis. Primary antibodies to MET, pTyr 1234/1235 MET, protein kinase B (PKB, AKT), pSer 473 AKT, signal transducer and activator of transcription $1 / 3$ (STAT1/3), phosphoSTAT1/3, MCL1 apoptosis regulator, BCL2 family member (MCL1), cyclin D3, caspase 3 and poly (ADP-ribose) polymerase (PARP) were obtained from Cell Signaling Technology (Danvers, MA, USA). Primary antibodies to $\beta$-actin were purchased from Sigma-Aldrich (St. Louis, MO, USA). Primary antibodies for B-cell lymphoma-extra large (BCL-xL), interleukin-6 (IL6), and jun proto-oncogene, AP-1 transcription factor subunit (c-JUN) were obtained from Santa Cruz Biotechnology (Santa Cruz, CA, USA). Primary antibodies were detected with corresponding horseradish peroxidase-conjugated goat anti-mouse or goat antirabbit secondary antibody (Cell Signaling Technology), and immunoreactive proteins were visualized using an Enhanced ChemiLuminescence kit or LAS4000 (GE Healthcare BioSciences, Chicago, IL, USA). The intensities of protein bands were calculated with Quantity One software (Bio-Rad, Hercules, CA, USA) for quantification.

Generation of patient-derived (PDX) models. All procedures for patient-derived primary cells and xenograft were approved by the Asan Medical Center Institutional Review Board (IRB\#2012-0112), and Asan Medical Center Institutional Animal Care and Use Committee. All experimental protocols were carried out in accordance with Asan Medical Center institutional guidelines for the care and use of animals. All patient tumor tissues were obtained with informed consent in accordance with the ethical standards of the Asan Medical Center Institutional Research Committee. Patientderived primary cells and mouse xenograft were established as described in previous reports (14-16). Briefly, a small piece of patient tumor tissues were minced and subsequently digested using $1 \mathrm{mg} / \mathrm{ml}$ of type IV collagenase (Sigma Chemical Co, St. Louis, MO, USA.) in Dulbecco's modified Eagle's medium/F12. After 90 min incubation at $37^{\circ} \mathrm{C}$, tissues were washed and plated with bronchiolar epithelial basal medium (Lonza, Walkersville, MD, USA) containing bovine pituitary extract, human epidermal growth factor, GA-1000, insulin and a Lonza REGM ${ }^{\mathrm{TM}}$ Renal Epithelial Cell Growth Medium SingleQuots ${ }^{\mathrm{TM}}$ Kit. For generation of PDX, a piece of tumor tissue in $3 \times 3 \times 3 \mathrm{~mm}^{3}$ size was implanted subcutaneously into flank of 6-week-old NOD-SCID mice (Charles River Laboratories, Wilmington, MA, USA). After 2-3 months, when the tumor size reached more than $1 \mathrm{~cm}^{3}$, the tumors were surgically removed and propagated by transplanting into male athymic nude mice (BALB/c-nude; 6 weeks old; Japan SLC, Hamamatsu, Japan) (14-16).

Assessment of tumor growth inhibition in vitro and in vivo. All procedures for NSCLC cell xenografts were approved by the Asan Medical Center Institutional Animal Care and Use Committee. Xenograft experiments were as described in previous reports (14-16). Briefly, H1975 cells $\left(2 \times 10^{6}\right)$ were injected subcutaneously into the flank of 6-week-old male athymic nude mice. Treatment was initiated when tumors in each group achieved an average volume of $100 \mathrm{~mm}^{3}$. The mice were divided into two treatment groups: one group treated five times with 2 or $10 \mathrm{mg} / \mathrm{kg}$ of taxotere every 5 days by intravenous injection, with phosphate-buffered saline as vehicle; and a control group was injected with phosphate-buffered saline alone. Tumor volumes were measured with caliper three times weekly and monitored for 25 days after the onset of treatment. The difference in tumor size was determined according to the formula: $\mathrm{W} 1 \times \mathrm{W} 2 \times \mathrm{W} 2 / 2$ $\mathrm{mm}^{3}$, where $\mathrm{W} 1$ was the largest tumor diameter and W2 the smallest. The PDX tumors were transplanted into male athymic nude mice and 


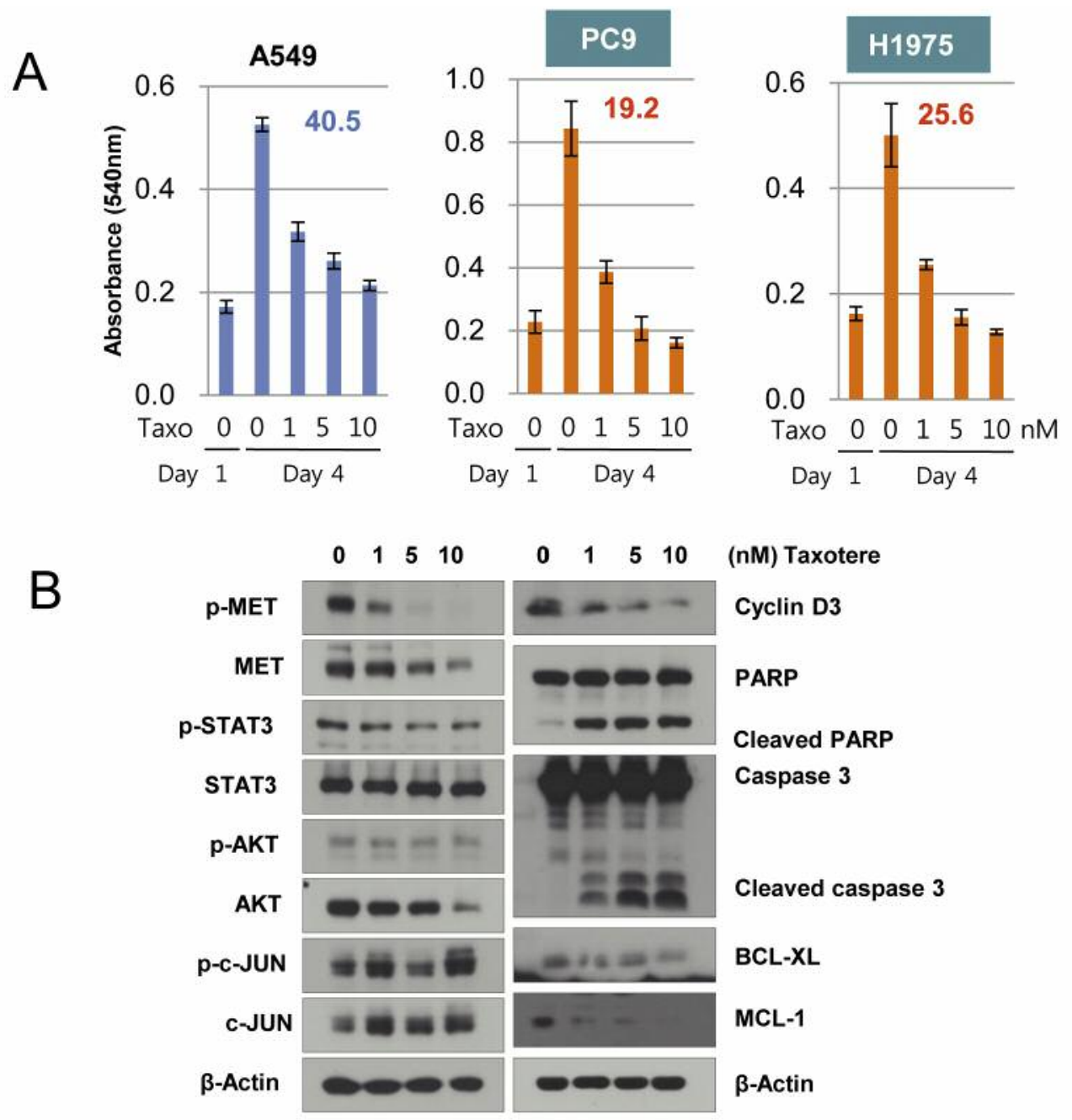

Figure 1. The effect of taxotere (Taxo) on non-small-cell lung carcinoma (NSCLC) cell growth. A: Cells were treated with increasing concentrations of taxotere for 3 days. Inhibitory effect of drug was determined as the cell growth ratio of 0 to $10 \mathrm{nM}$ taxotere treatment on day 4. B: Expression of phosphor-MET proto-oncogene, receptor tyrosine kinase (p-MET), phosphor-signal transducer and activator of transcription 3 (p-STAT3), phosphor-AKT ( $p$-AKT), phosphor-jun proto-oncogene, AP-1 transcription factor subunit (p-c-JUN), poly-ADP ribose polymerase (PARP), B-cell lymphoma-extra large (BCL-xL) and MCL1 apoptosis regulator, BCL2 family member (MCL1) proteins in H1975 NSCLC cells were evaluated after 3 days of treatment with taxotere.

grown to an average $100 \mathrm{~mm}^{3}$, and used for in vivo efficacy analysis as xenograft experiments with lung cancer cells.

Cell growth inhibition. Lung cancer cells or patient-derived cultured lung primary cells were treated with different concentrations of 1 to $10 \mathrm{nM}$ of taxotere with and without 1 to $2 \mu \mathrm{M}$ of crizotinib in appropriate media for 24 to $72 \mathrm{~h}$. Cell proliferation was measured with the 3-(4,5-dimethylthiazol-2-yl)-2,5-diphenyltetrazolium bromide (MTT; Sigma) assay as previously described (17).

Quantitative reverse transcriptional-polymerase chain reaction ( $R T$ $P C R)$. RT-PCR was carried out using a High Capacity cDNA Reverse Transcription kit (Applied Biosystems). Primers in Table I used for the experiments were obtained from Macrogen Inc. (Seoul,
Republic of Korea). Three independent experiments were performed to confirm gene down-regulation (17).

Statistical analysis. Data are expressed as the means \pm SD of triplicate samples or at least three independent experiments. Statistical significance was determined with Student's $t$-test with threshold of $p<0.05$.

\section{Results}

Taxotere reduced cell proliferation of NSCLC cells including cells with acquired TKI resistance. We examined efficiency of taxotere on NSCLC cells as potential 


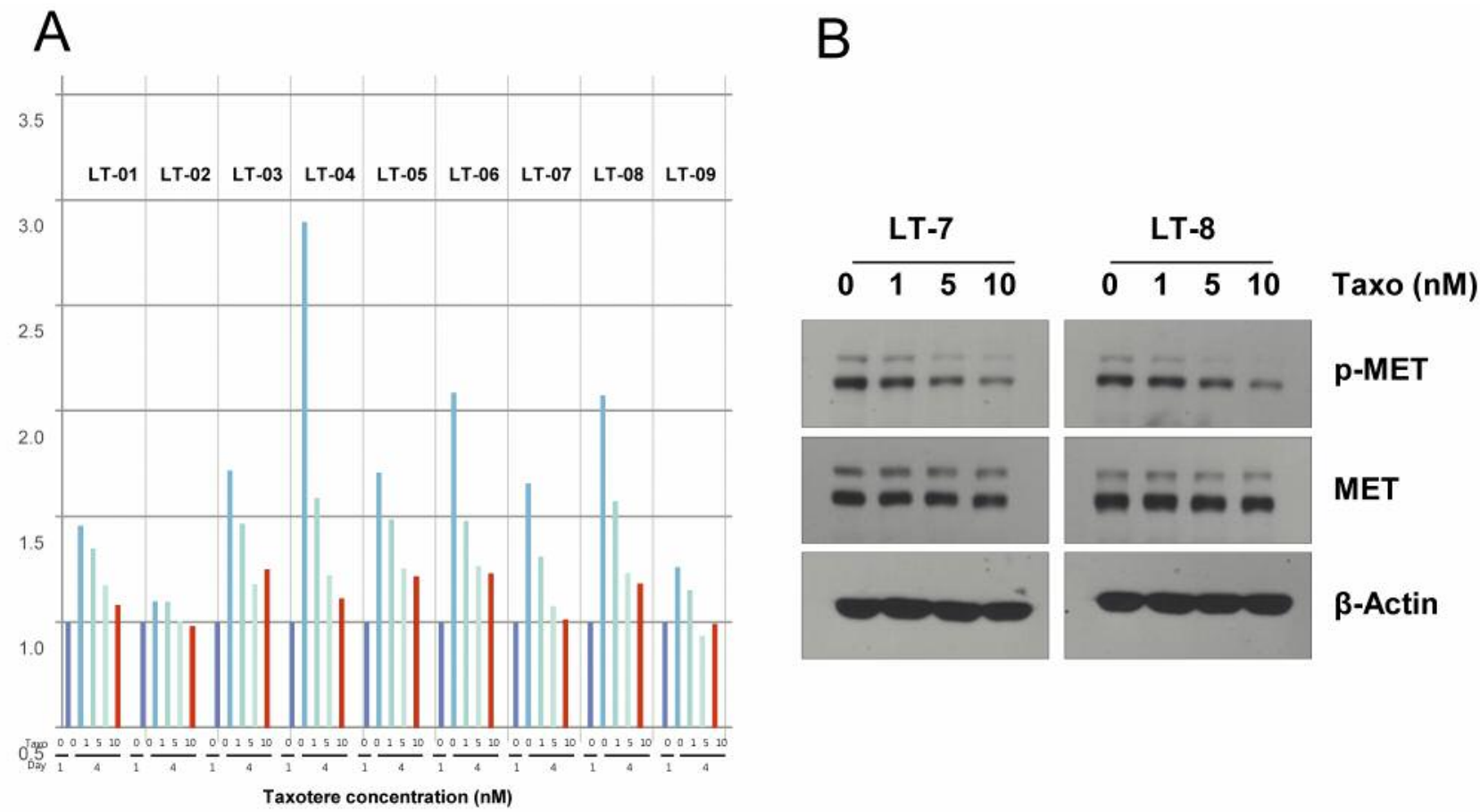

Figure 2. A: The effect of taxotere (Taxo) on growth of multiple patient-derived primary lung tumor (LT) cells. Cells were treated with increasing concentrations of taxotere for 3 days. B: Expression of phosphor-MET proto-oncogene, receptor tyrosine kinase (p-MET), phosphor-signal transducer and activator of transcription 3 ( $p$-STAT3), phosphor-AKT ( $p$-AKT), and poly-ADP ribose polymerase (PARP) proteins in cells after 3 days of treatment at the indicated concentration of taxotere.

therapeutics against cells with acquired resistance to TKI. Interestingly, cells with activated EGFR signaling, thereby TKI-sensitive cells (PC9), as well as TKI-resistant cells such as EGFR T790M mutant cells (H1975) were also responsive to the drugs; $\mathrm{PC} 9$ and $\mathrm{H} 1975$ showed dramatic growth inhibition, as low as $19 \%$ and $26 \%$, respectively after 3 days of drug treatment (Figure 1A). These results indicate that taxotere is effective in cell growth inhibition regardless of EGFR activation or TKI resistance.

Taxotere inhibited phosphor-MET expression on cells with acquired TKI resistance. By binding to tubulin followed by producing microtubules, taxotere results in cell growth inhibition and apoptosis. However, there is no report relevant to MET signaling pathway, which affects tumor cell growth and metastasis. We analyzed expression of proteins associated with survival signaling pathways in H1975 cells, harboring EGFR T790M and activated MET, after taxotere treatment. Taxotere efficiently reduced phosphorylated MET on H1975 cells, and phosphorylated STAT3 was least reduced at the highest concentration of the drug. Phosphorylation of other proteins including AKT and p38, as well as c-JUN, was not affected by drug treatment. Taxotere also induced PARP and caspase- 3 cleavage and the reduction of cyclin D3 and MCL1, indicating apoptosis and cell-cycle arrest occurred after drug treatment, but there was no effect on the expression of BCL-XL (Figure 1B) or survivin (data not shown).

Figure 3. The effect of taxotere on tumor growth in H1975 non-smallcell lung carcinoma cell xenografts. A: Mice inoculated with $H 1975$ cells were treated intravenously with phosphate-buffered saline (untreated) or $10 \mathrm{mg} / \mathrm{kg}$ taxotere (treated) five times once every 5 days. Tumor volume was calculated using the formula for an ellipsoid sphere. Independent xenograft experiments were performed three times. B: Average tumor size of treated and untreated groups. ***Significantly different at $p<0.001$. C: Tumors at the end point of the experiment are presented. D: Body weights of mice were measured to monitor the sideeffects of the drug. E: Expression of proteins, phosphor-MET protooncogene, receptor tyrosine kinase (p-MET), phosphor-signal transducer and activator of transcription 3 ( $p$-STAT3), phosphor-AKT (p-AKT), poly-ADP ribose polymerase (PARP), MCL1 apoptosis regulator, BCL2 family member (MCL1) and interleukin-6 (IL6), were analyzed by western blotting. F: Quantification of the expression of each protein was compared to that of $\beta$-actin expression. Significantly different at $* p<0.05, * * p<0.01$ and $* * * p<0.005$. 
A

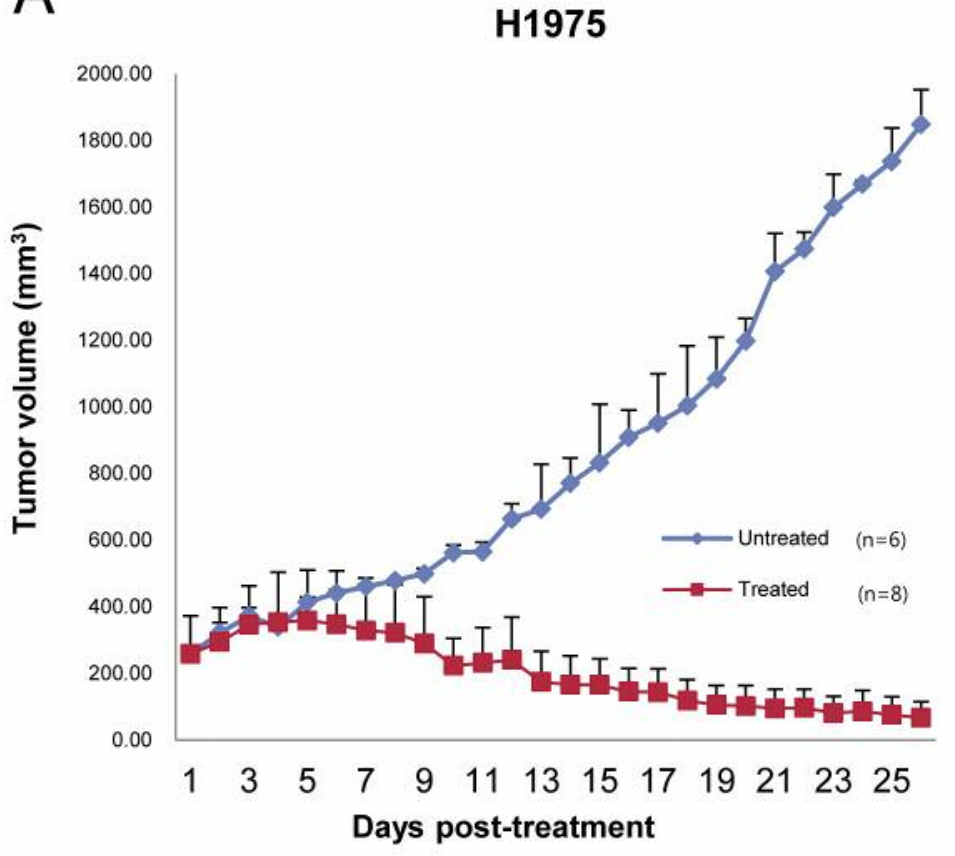

B

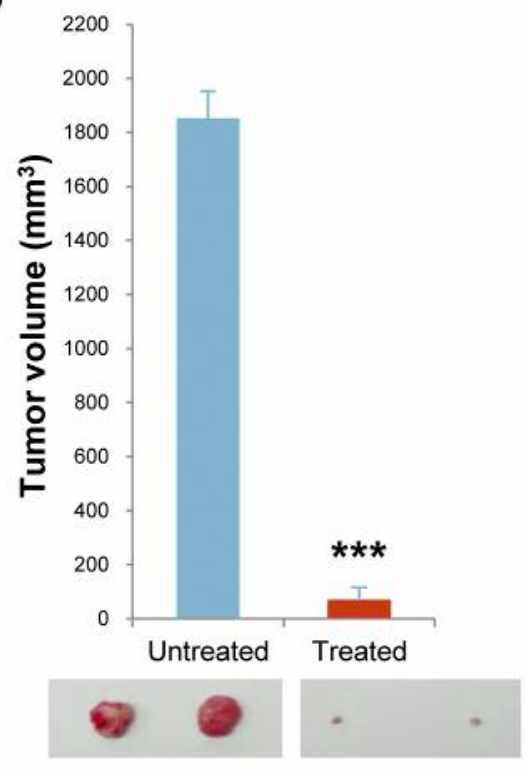

D

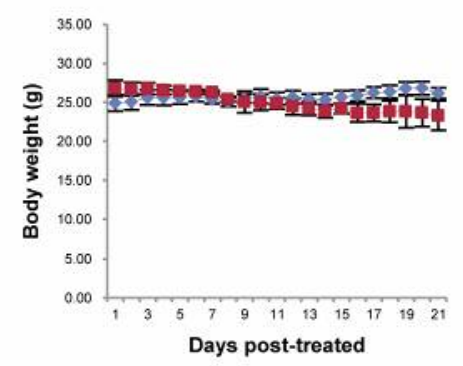

$E$
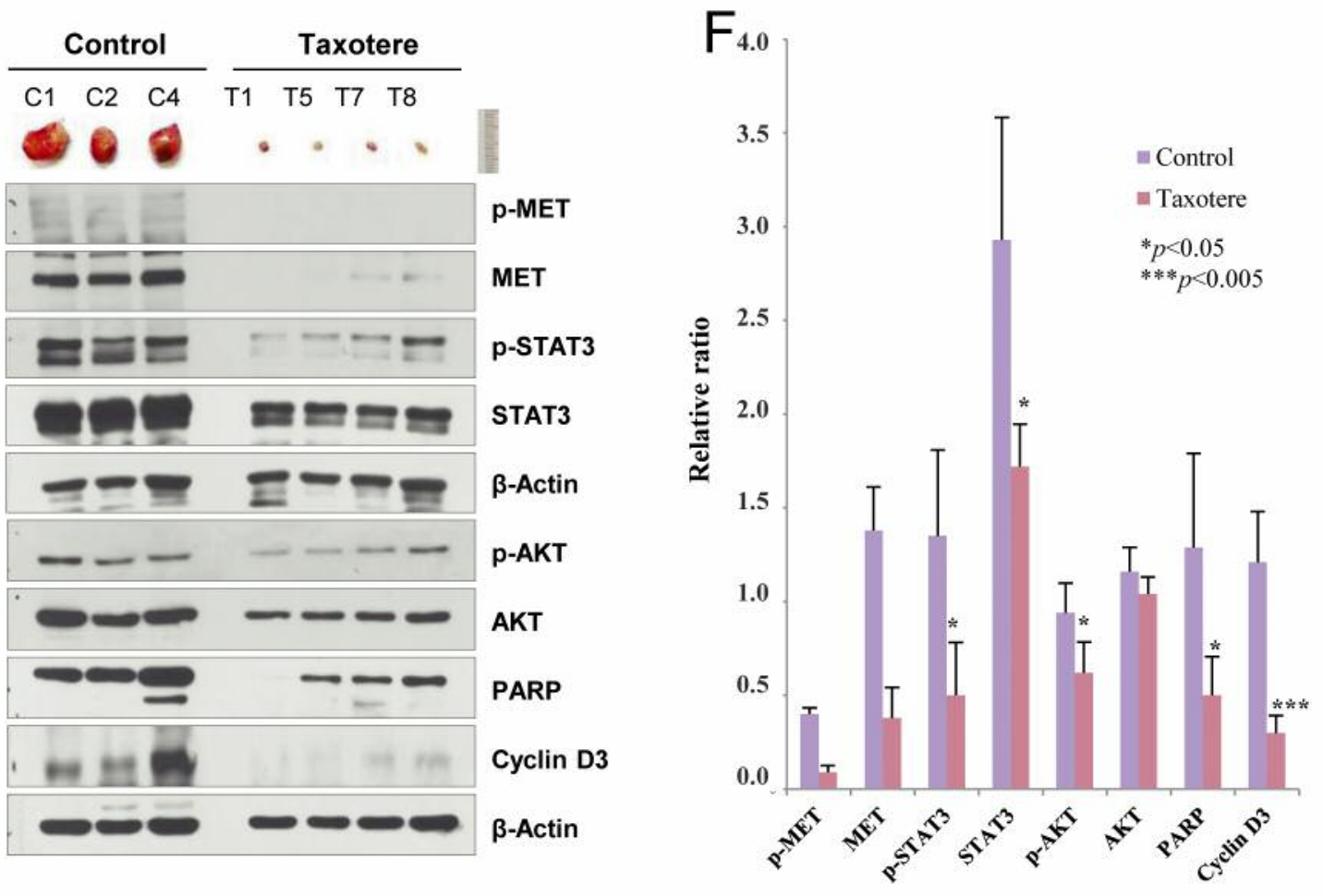

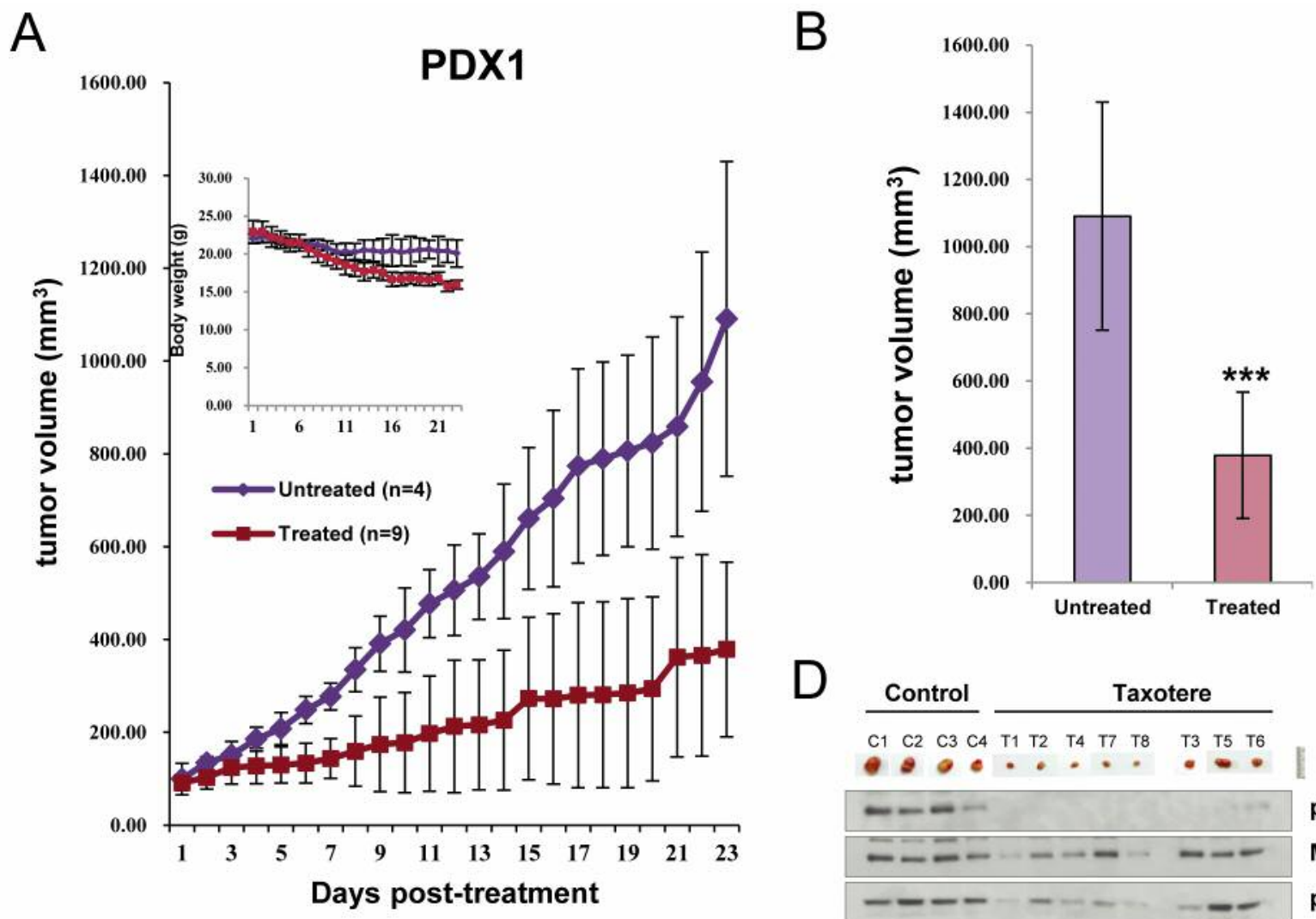

D

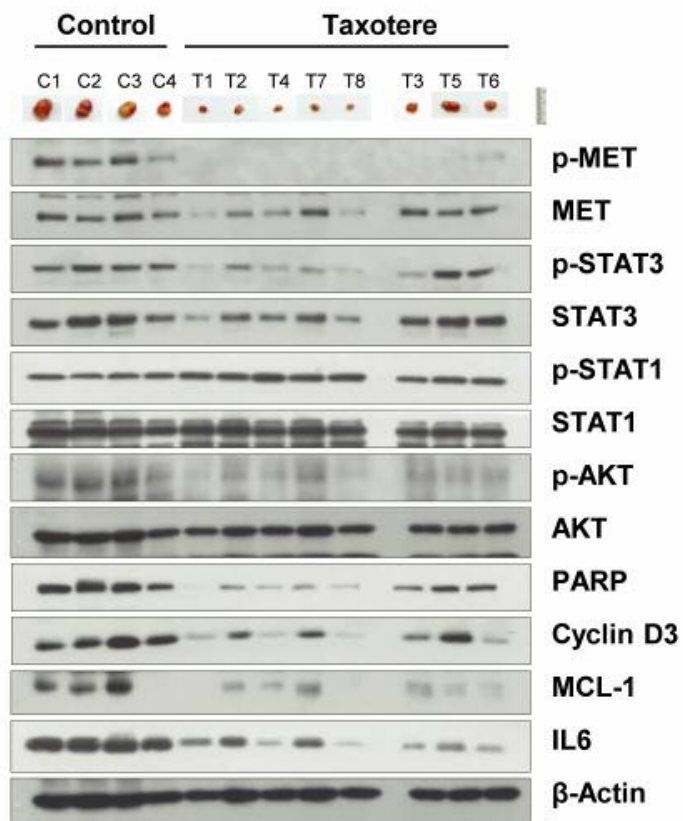

Figure 4. Effect of taxotere on tumor growth in mice with patient-derived xenografts (PDX). A: Mice transplanted with PDX1 tumors were treated intravenously with phosphate-buffered saline (vehicle) or $10 \mathrm{mg} / \mathrm{kg}$ taxotere five times once every 5 days. Tumor volume was calculated using the formula for an ellipsoid sphere. Independent xenograft experiments were performed three times. Body weights are depicted in the inset chart. B: Average tumor size of treated and untreated groups. C: Tumors collected at the end point of the experiment. D: Expression of proteins, phosphorMET proto-oncogene, receptor tyrosine kinase (p-MET), phosphor-signal transducer and activator of transcription 3 (p-STAT3), were analyzed by western blotting. $* * *$ Significantly different at $p<0.005$.

Taxotere reduced proliferation of lung PDCs expressing a high level of MET. Patient-derived models have been developed to recapitulate patient status and are beneficially utilized for cancer research. We evaluated the effect of taxotere on cultured lung PDCs expressing a high level of phosphorylated MET. The primary cell growth was reduced to more than 50\% in PDCs (LT-4, LT-6, LT-7, LT-8) among tested after 3 days of taxotere treatment (Figure 2A). Phosphorylated MET was reduced on LT-7 and LT-8 PDCs (Figure 2B). Taken together, these findings show taxotere reduced tumor cell growth, partially affecting on MET signaling. 


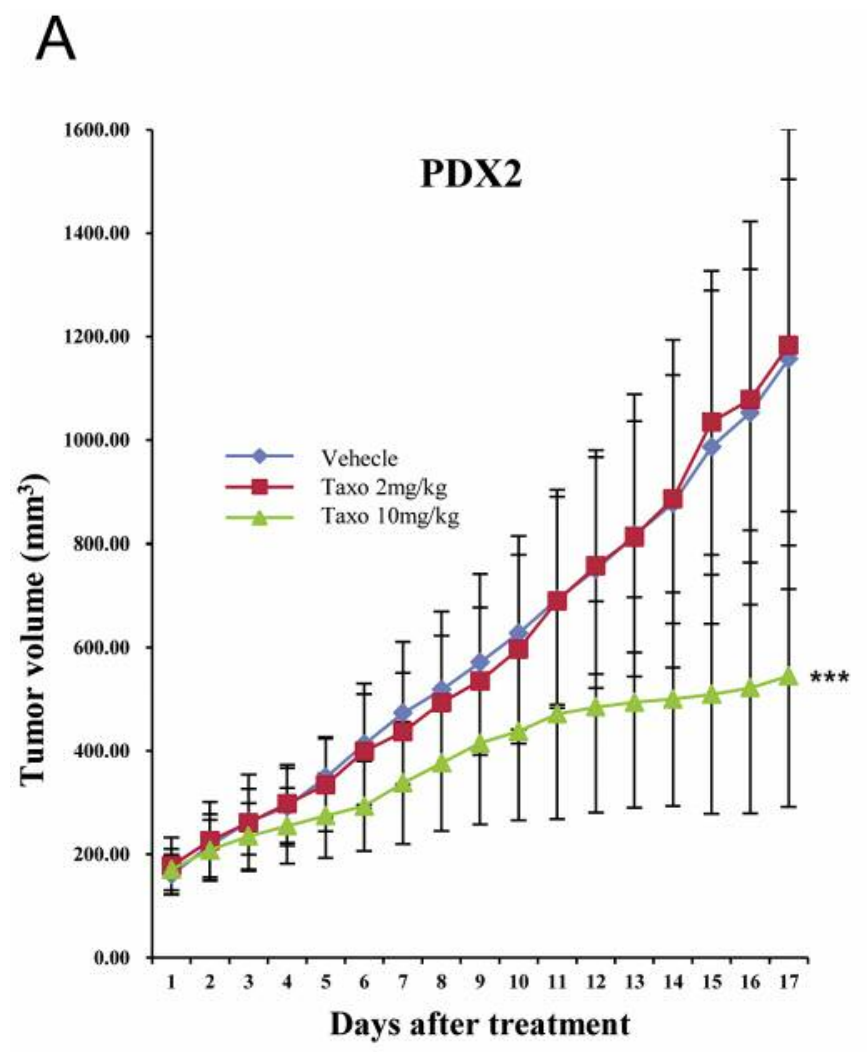

B

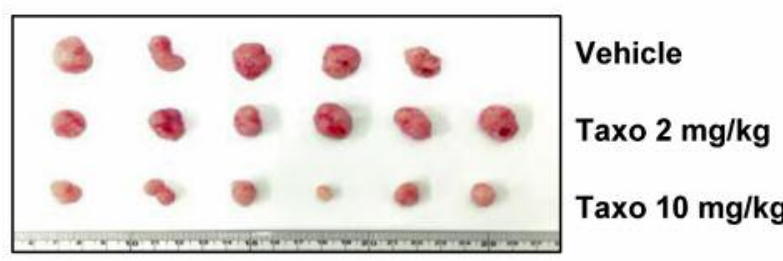

C
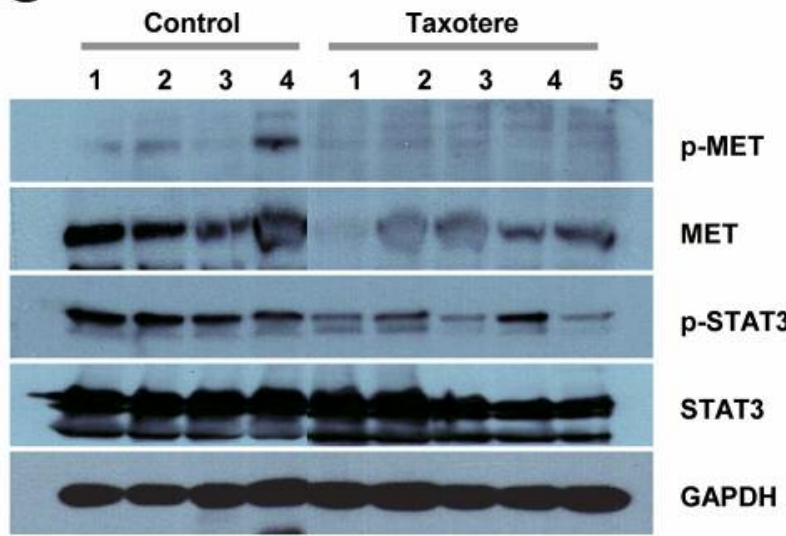

Figure 5. Effect of taxotere on tumor growth in patient-derived xenografts (PDX) in mice. A: Mice transplanted with PDX2 tumors were treated intravenously with phosphate-buffered saline (vehicle), 2 or $10 \mathrm{mg} / \mathrm{kg}$ taxotere five times once every 5 days. Tumor volume was calculated using the formula for an ellipsoid sphere. Independent xenograft experiments were performed three times. ***Significantly different at $p=0.009$. B: The tumors from phosphate-buffered saline (untreated) or taxotere (treated) mice are presented.C: Expression of MET proto-oncogene, receptor tyrosine kinase (MET) and signal transducer and activator of transcription 3 (STAT3), glyceraldehyde 3-phosphate dehydrogenase (GAPDH) were analyzed by western blotting.

Taxotere reduced tumor growth of TKI-refractory NSCLC xenograft models. The efficacy of taxotere was evaluated in vivo using the H1975 xenograft model. Tumor growth was dramatically affected with no significant body weight changes (Figure 3A-D). The expression of phosphorylated MET was reduced in remnant tumors of drug treated mice. The reduction of PARP and cyclin D3 expression indicated that cell death through apoptosis and cell-cycle arrest occurred after drug treatment. The expression of phosphorylated MET was reduced (Figure $3 \mathrm{E}$ and F). These results suggest that MET signaling may also be involved in the mechanism of tumor regression reduced by taxotere.

Taxotere reduced tumor growth of PDX model with high expression of MET. Using PDX models that faithfully and accurately reflects the patients' tumors with regard to histopathology, genetics and therapeutic response, we evaluated the effect of taxotere on tumor growth and MET activation in vivo. PDX1 tumors from adenocarcinoma tissue that expressed phosphorylated MET were shrunken to approximately $40 \%$ after treatment with taxotere (Figure 4AC). Reduction of tumor size was accompanied by reduced expression of proteins on phosphor-MET, phosphor-STAT3 and IL6, implicating the role of these proteins in tumor growth and the effect on the MET--STAT3-IL6 axis (Figure 4D). The reduction of PARP, cyclin D3 and MCL1 expression was also detected on drug-treated tumors, indicating apoptosis and cell-cycle arrest. Taxotere treatment also reduced tumor growth to less than 50\% of PDX2 tumors from lung adenocarcinoma tissue compared to untreated tumors (Figure 5A and B). The expression of phosphor-MET and phosphor-STAT3 proteins were decreased in tumors treated with taxotere (Figure 5C). These results support the notion that taxotere suppressed tumor growth through inhibiting MET signaling pathways.

MET inhibitor compensated taxotere effect in NSCLC cell growth. Since taxotere reduced the level of phosphorylated 
A
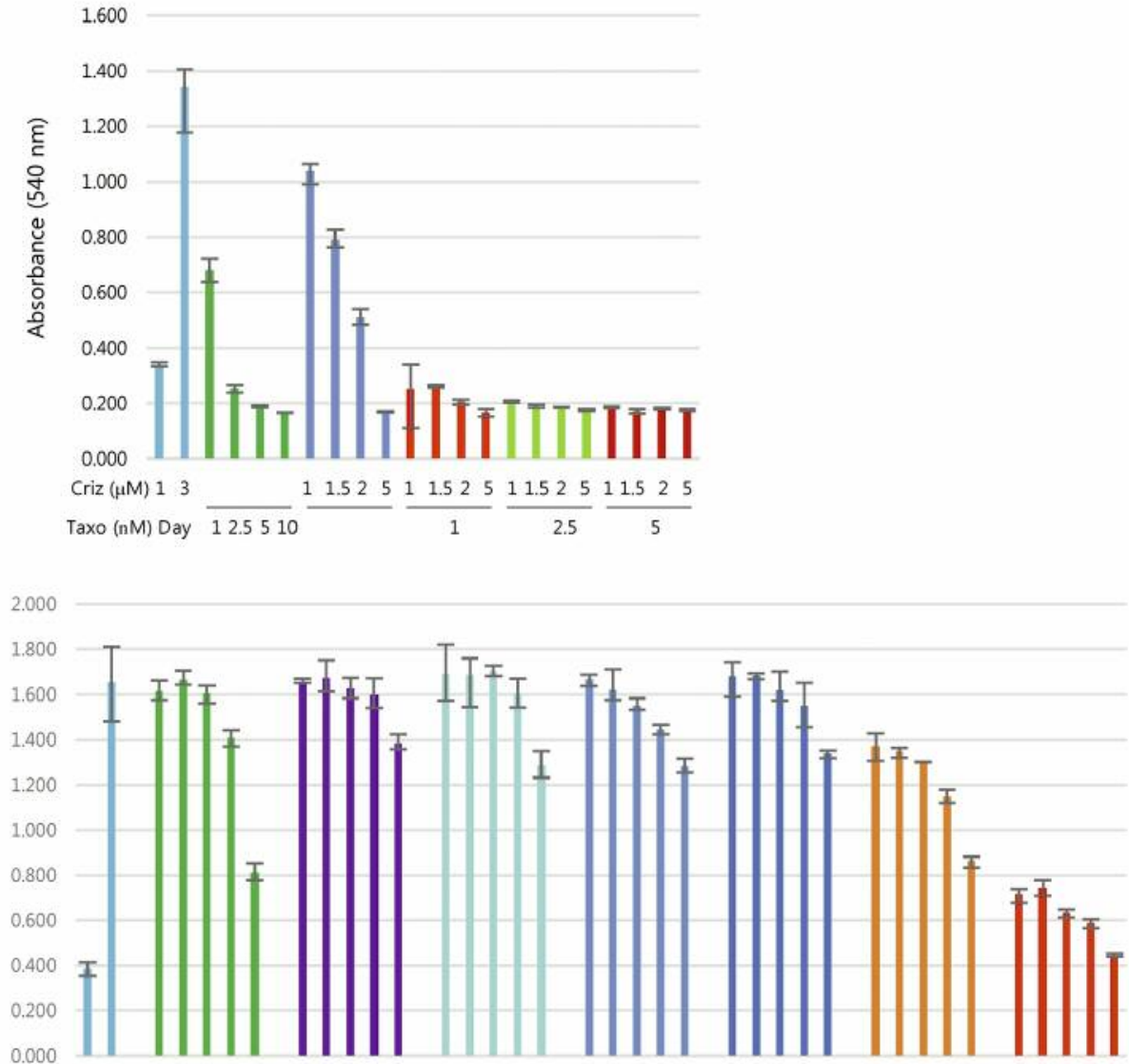

Criz $(\mu M) \quad 13$

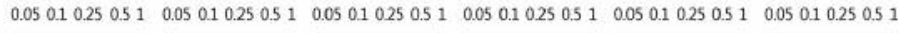

Taxo $(\mathrm{nM}) \quad$ Day 0.050 .10 .250 .5

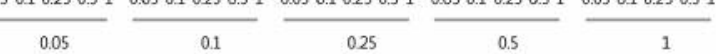

B
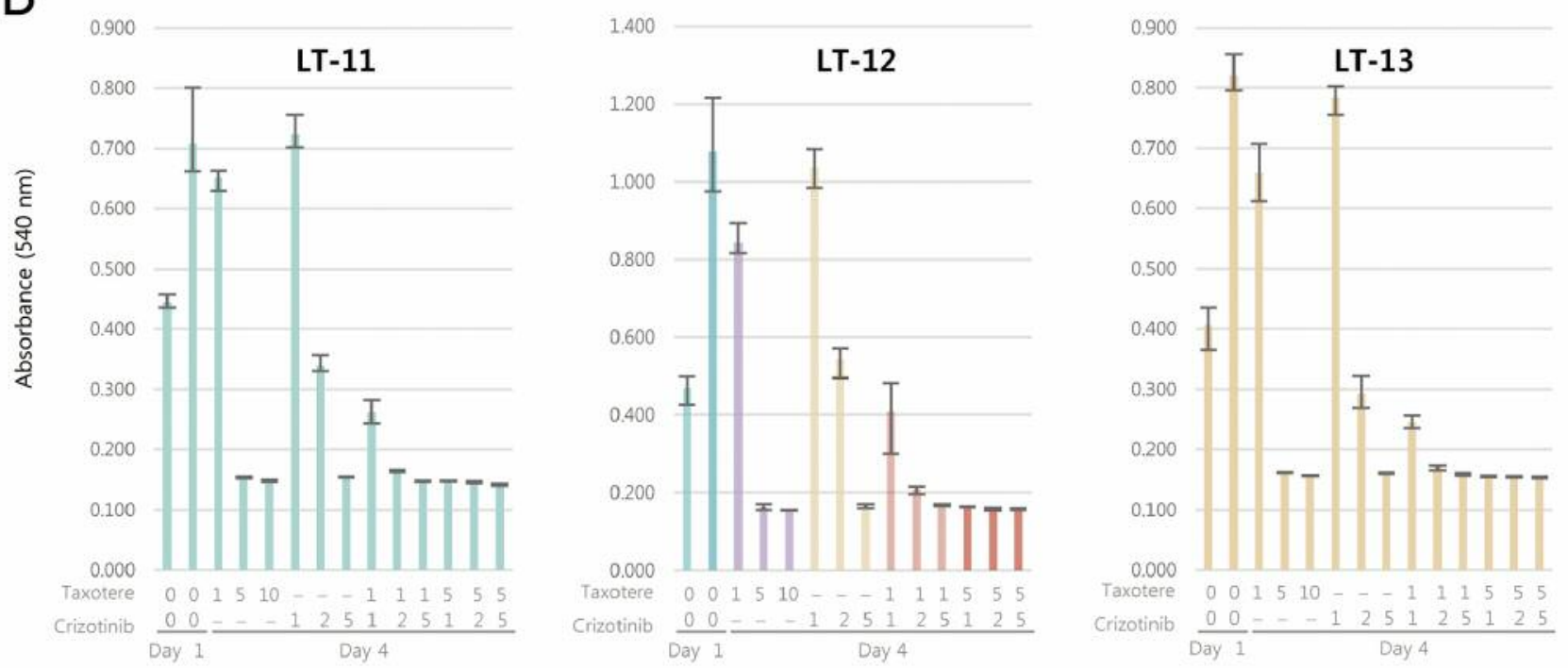

Figure 6. The effect of taxotere (Taxo) and MET proto-oncogene, receptor tyrosine kinase (MET) inhibitor crizotinb (Criz) on growth. A: Cell proliferation of $H 1975$ was determined under different treament with combinations of increasing concentrations of taxotere and crizotinb for 3 days. B: Cell growth-inhibitory effects on patient-derived cancer cells. 
Table II. Patients' tumor information established as patient-derived cancer cells (LT) or patient-derived xenograft (PDX).

\begin{tabular}{lcccc}
\hline No. & Gender & Age, years & Histological diagnosis & Differentiation \\
\hline LT-01 & F & 59 & Sarcomatoid carcinoma & - \\
LT-02 & F & 61 & Adenocarcinoma & Moderately differentiated \\
LT-03 & M & 72 & Sarcomatoid carcinoma & - \\
LT-04 & M & 71 & Adenocarcinoma & Moderately differentiated \\
LT-05 & F & 60 & Squamous cell carcinoma & Moderately differentiated \\
LT-06 & M & 54 & Adenocarcinoma & Moderately differentiated differentiated \\
LT-07 & M & 58 & Adenocarcinoma & Moderately differentiated \\
LT-08 & F & 60 & Adenocarcinoma & Moderately differentiated \\
LT-09 & F & 72 & Adenocarcinoma & Poorly differentiated \\
LT-11 & M & 58 & Squamous cell carcinoma & Moderately differentiated \\
LT-12 & M & 65 & Adenocarcinoma & Moderately differentiated \\
LT-13 & M & 46 & Adenocarcinoma & Poorly differentiated \\
PDX1 & M & 72 & Adenocarcinoma & Poorly differentiated \\
PDX2 & F & 66 & &
\end{tabular}

F: Female; M: male.

MET in H1975 in vitro as well as in vivo treatments, we evaluated the additive/synergistic effect of taxotere with MET inhibitor crizotinib on tumor cell growth. Combinational treatment of $1 \mathrm{nM}$ taxotere and $1 \mu \mathrm{M}$ crizotinib dramatically reduced cell growth to less than $20 \%$, and $0.5 \mathrm{nM}$ taxotere and $1 \mu \mathrm{M}$ crizotinib decreased cell proliferation to approximately $50 \%$ (Figure 6A) in H1975 cells, implicating that the role of MET signaling pathway in the cell growth. Three days of cotreatment of $1 \mathrm{nM}$ taxotere with $1 \mu \mathrm{M}$ crizotinib reduced cell growth to 30 to $40 \%$ in PDCs (Figure 6B). Phosphorylated MET expression was barely detected, but the level of cleaved PARP was increased in H1975 cells treated with taxotere or taxotere plus crizotinib (Figure 7A). Co-treatment of taxotere and crizotinib for $24 \mathrm{~h}$ synergistically resulted in reduction of IL6 and C-C motif chemokine ligand 26 (CCL26) expression, and the expression of IL8 was reduced on taxotere treatment (Figure 7B) in semi-quantitative RT-PCR assay. These results together imply that taxotere would be a candidate chemotherapeutic for EGFR inhibitor-refractory cells through halting MET signaling.

\section{Discussion}

Precision cancer medicine akin to targeted therapeutics is the most desired treatment for patients with cancer, including those with lung cancer $(1,18,19)$. One of the obstacles to pursuing targeted therapy is the development of acquired resistance to drugs. To combat resistance and improve the therapeutic effect, combinational clinical trials are attempted using targeted agents plus traditional chemotherapeutics. In this study, we evaluated taxotere efficiency in inhibiting tumor growth of cells exhibiting resistance to EGFR inhibitor or expressing activated MET. Its efficacy was verified on multiple models, especially

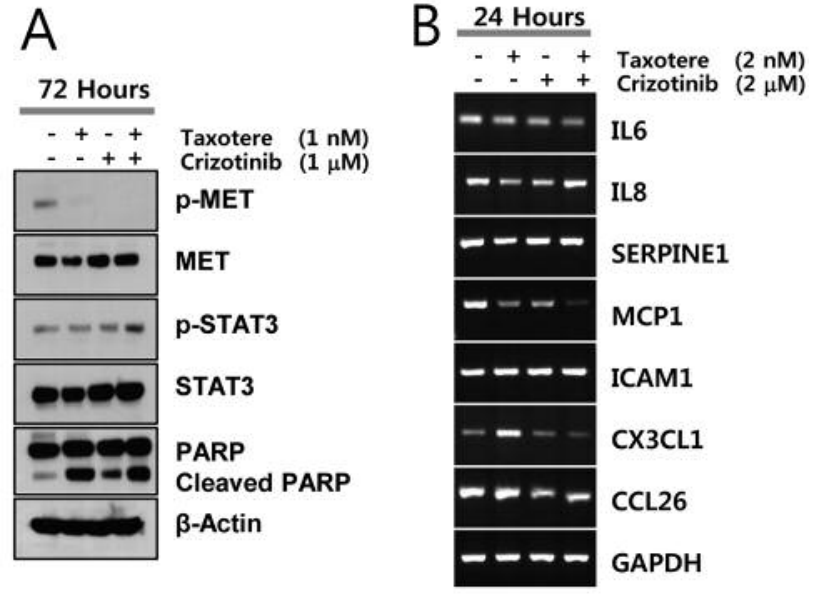

Figure 7. The effect of taxotere and MET inhibitor crizotinb on $H 1975$ cells. A: Expression of proteins, phosphor-MET proto-oncogene, receptor tyrosine kinase (MET), phosphor-signal transducer and activator of transcription 3 ( $p$-STAT3), poly-ADP ribose polymerase (PARP), in H1975 non-small cell lung cancer cells were evaluated after 3 days of treatment with taxotere with and without crizotinib. B: Chemokines/cytokines, interleukin-6 (IL6), C-C motif chemokine ligand 2 (monocyte chemoattractant protein 1, MCP1), intercellular adhesion molecule 1 (ICAM1), C-X3-C motif chemokine ligand (CX3CL1), C-C motif chemokine ligand 26 (CCL26) and glyceraldehyde 3-phosphate dehydrogenase (GAPDH) affected by taxotere and crizotinib were confirmed by reverse transcription polymerase chain reaction analysis.

on PDCs and PDXs. We also showed that combinational treatment of taxotere and MET inhibitor provided an additive inhibitory effect on tumor growth, implicating the possibility to lessen the side-effects of chemotherapeutics by allowing 
dose reduction. Additionally, the decrease in expressions of IL6, IL8 and CCL2 were discovered, coincidently with cell proliferation or tumor shrinking, suggesting their involvement of tumor microenvironment.

The amplification of MET is reported as one of resistance mechanism to EGFR inhibitors in lung cancer patients. Osimertinib (Tagrisso) targeting T790M secondary mutation at $E G F R$, has been approved as first-line treatment for advanced NSCLC in 2015 (20). However, increasing evidence has also revealed acquired resistance to this drug as a growing clinical challenge that is poorly understood. Strategies to overcome diverse resistance to targeted drugs are urgently required, and combination treatment and re-evaluation of standard chemotherapeutics may be solutions. When the efficiency of combinational treatments was explored with EGFR inhibitor, cetuximab or panitumumab, and chemotherapeutics, such as cisplatin or docetaxel in NSCLC cells, including H1975, augmented tumor regression was noted $(13,21)$.

The contributions of chemokines and cytokines to tumorigenesis are not fully understood, although they have emerged as relevant molecules in shaping the tumor microenvironment (22). The regulation of IL6 and IL8 through STAT1/3 has been investigated and is interconnected with other signaling network such as a mitogen-activated protein kinase, phosphatidylinositol 3-kinase and nuclear factor kappa-lightchain-enhancer of activated B-cells pathways. Taken together, it would be worth to elucidate the role of MET signaling in the tumor microenvironment and in progression, especially in regulation of chemokine/cytokine expression, upon treatment with cancer therapeutics. As a promising adjuvant candidate in targeting against MET signaling, further investigation should elucidate the detailed mechanism in terms of the immune microenvironment.

\section{Conflicts of Interest}

All Authors declare no competing interests.

\section{Authors' Contributions}

Hyemin Mun, Sun-Hye Lee, Se-Young Jo, Ju-Hee Oh, Areum Lee and Bora Lee: Experimental studies, data acquisition, statistical analysis, and article review. Chu-Hee Lee: Study design, experimental design, data analysis, statistical analysis, article preparation and review. Si Jin Jang: Study and experimental support. Young-Ah Suh: Study design, data analysis, statistical analysis, article preparation, review and editing. All Authors read and approved the final article.

\section{Acknowledgements}

This study was funded by a grant from the National Research Foundation of Korea Grant funded by the Korean Government (NRF-2017R1A2B4012110). Authors would like to thank to Dr.
Heekyung K. Chae, Department of Pediatrics at University of California San Diego School of Medicine for discussion on medical viewpoints, writing comments and corrections on article.

\section{References}

1 Vargas AJ and Harris CC: Biomarker development in the precision medicine era: Lung cancer as a case study. Nat Rev Cancer 16(8): 525-537, 2016. PMID: 27388699. DOI: 10.1038/nrc.2016.56

2 Clohessy JG and Pandolfi PP: The mouse hospital and its integration in ultra-precision approaches to cancer care. Front Oncol 8: 340, 2018. PMID: 30211119. DOI: $10.3389 /$ fonc. 2018.00340

3 Castellanos EH and Horn L: Generations of epidermal growth factor receptor tyrosine kinase inhibitors: Perils and progress. Curr Treat Options Oncol 16(10): 51, 2015. PMID: 26364032. DOI: $10.1007 / \mathrm{s} 11864-015-0365-1$

4 Bertotti A and Sassi F: Molecular pathways: Sensitivity and resistance to anti-EGFR antibodies. Clin Cancer Res 21(15): 3377-3383, 2015. PMID: 26071484. DOI: 10.1158/10780432.CCR-14-0848

5 Sequist LV, Waltman BA, Dias-Santagata D, Digumarthy S, Turke AB, Fidias P, Bergethon K, Shaw AT, Gettinger S, Cosper AK, Akhavanfard S, Heist RS, Temel J, Christensen JG, Wain JC, Lynch TJ, Vernovsky K, Mark EJ, Lanuti M, Iafrate AJ, Mino-Kenudson $M$ and Engelman JA: Genotypic and histological evolution of lung cancers acquiring resistance to EGFR inhibitors. Sci Transl Med 3(75): 75ra26, 2011. PMID: 21430269. DOI: 10.1126/scitranslmed.3002003

6 Wangari-Talbot $\mathrm{J}$ and Hopper-Borge E: Drug resistance mechanisms in non-small cell lung carcinoma. J Can Res Updates 2(4): 265-282, 2013. PMID: 24634705. DOI: 10.6000/1929-2279.2013.02.04.5

7 Bean J, Brennan C, Shih JY, Riely G, Viale A, Wang L, Chitale D, Motoi N, Szoke J, Broderick S, Balak M, Chang WC, Yu CJ, Gazdar A, Pass H, Rusch V, Gerald W, Huang SF, Yang PC, Miller V, Ladanyi M, Yang $\mathrm{CH}$ and Pao W: Met amplification occurs with or without T790M mutations in EGFR-mutant lung tumors with acquired resistance to gefitinib or erlotinib. Proc Natl Acad Sci USA 104(52): 20932-20937, 2007. PMID: 18093943. DOI: 10.1073/pnas.0710370104

8 Turke AB, Zejnullahu K, Wu YL, Song Y, Dias-Santagata D, Lifshits E, Toschi L, Rogers A, Mok T, Sequist L, Lindeman NI, Murphy C, Akhavanfard S, Yeap BY, Xiao Y, Capelletti M, Iafrate AJ, Lee C, Christensen JG, Engelman JA and Janne PA: Preexistence and clonal selection of MET amplification in EGFR mutant NSCLC. Cancer Cell 17(1): 77-88, 2010. PMID: 20129249. DOI: $10.1016 /$ j.ccr.2009.11.022

9 Cecchi F, Rabe DC and Bottaro DP: Targeting the HGF/MET signaling pathway in cancer therapy. Expert Opin Ther Targets 16(6): 553-572, 2012. PMID: 22530990. DOI: 10.1517/14728222.2012.680957

10 Mo HN and Liu P: Targeting MET in cancer therapy. Chronic Dis Transl Med 3(3): 148-153, 2017. PMID: PMC5643781. DOI: $10.1016 /$ j.cdtm.2017.06.002

11 Mok TS and Loong HH: Are we ready for immune checkpoint inhibitors for advanced non-small-cell lung cancer? Lancet 387(10027): 1488-1490, 2016. PMID: 26712085. DOI: $10.1016 / \mathrm{S} 0140-6736(15) 01308-2$ 
12 Nagarsheth N, Wicha MS and Zou W: Chemokines in the cancer microenvironment and their relevance in cancer immunotherapy. Nat Rev Immunol 17(9): 559-572, 2017. PMID: 28555670. DOI: 10.1038/nri.2017.49

13 Ornstein DL and Rigas JR: Taxotere: Clinical trials in non-small cell lung cancer. Oncologist 3(2): 86-93, 1998. PMID: 10388089 .

14 Seol HS, Suh YA, Ryu YJ, Kim HJ, Chun SM, Na DC, Fukamachi H, Jeong SY, Choi EK and Jang SJ: A patientderived xenograft mouse model generated from primary cultured cells recapitulates patient tumors phenotypically and genetically. J Cancer Res Clin Oncol 139(9): 1471-1480, 2013. PMID: 23817694. DOI: 10.1007/s00432-013-1449-6

15 Seol HS, Kang HJ, Lee SI, Kim NE, Kim TI, Chun SM, Kim TW, Yu CS, Suh YA, Singh SR, Chang S and Jang SJ: Development and characterization of a colon PDX model that reproduces drug responsiveness and the mutation profiles of its original tumor. Cancer Lett 345(1): 56-64, 2014. PMID: 24333725. DOI: 10.1016/j.canlet.2013.11.010

16 Shin SH, Park SS, Ju EJ, Park J, Ko EJ, Hwang JJ, Suh YA, Jang SJ, Lee JS, Ko BK, Kim KT, Lee JS, Song SY, Jeong SY and Choi EK: Establishment of a patient-derived xenograft for development of personalized HER2-targeting therapy in gastric cancer. Anticancer Res 38(1): 287-293, 2018. PMID: 29277785. DOI: 10.21873 /anticanres.12220

17 Lee SH, Lee JY, Jung CL, Bae IH, Suh KH, Ahn YG, Jin DH, Kim TW, Suh YA and Jang SJ: A novel antagonist to the inhibitors of apoptosis (IAPS) potentiates cell death in EGFRoverexpressing non-small-cell lung cancer cells. Cell Death Dis 5: e1477, 2014. PMID: 25321484. DOI: 10.1038/cddis.2014.447
18 Rubin MA: Health: Make precision medicine work for cancer care. Nature 520(7547): 290-291, 2015. PMID: 25877189. DOI: $10.1038 / 520290 \mathrm{a}$

19 Pasche B and Grant SC: Non-small cell lung cancer and precision medicine: A model for the incorporation of genomic features into clinical trial design. JAMA 311(19): 1975-1976, 2014. PMID: 24846033. DOI: 10.1001/jama.2014.3742

20 Janne PA, Yang JC, Kim DW, Planchard D, Ohe Y, Ramalingam SS, Ahn MJ, Kim SW, Su WC, Horn L, Haggstrom D, Felip E, Kim JH, Frewer P, Cantarini M, Brown KH, Dickinson PA, Ghiorghiu S and Ranson M: Azd9291 in EGFR inhibitor-resistant non-small-cell lung cancer. N Engl J Med 372(18): 1689-1699, 2015. PMID: 25923549. DOI: 10.1056/NEJMoa1411817

21 Steiner P, Joynes C, Bassi R, Wang S, Tonra JR, Hadari YR and Hicklin DJ: Tumor growth inhibition with cetuximab and chemotherapy in non-small cell lung cancer xenografts expressing wild-type and mutated epidermal growth factor receptor. Clin Cancer Res 13(5): 1540-1551, 2007. PMID: 17332300. DOI: 10.1158/1078-0432.CCR-06-1887

22 Vyas D, Laput $G$ and Vyas AK: Chemotherapy-enhanced inflammation may lead to the failure of therapy and metastasis. Onco Targets Ther 7: 1015-1023, 2014. PMID: 24959088. DOI: 10.2147/OTT.S60114

Received November 13, 2019

Revised November 29, 2019

Accepted December 2, 2019 